\title{
Production of Energetic Light Fragments in Spallation Reactions
}

\author{
Stepan G. Mashnik ${ }^{1, a}$, Leslie M. Kerby ${ }^{1,2}$, Konstantin K. Gudima ${ }^{3}$, and Arnold J. Sierk ${ }^{1}$ \\ ${ }^{1}$ Los Alamos National Laboratory, Los Alamos, NM 87545, USA \\ ${ }^{2}$ University of Idaho, Moscow, Idaho 83844-4264, USA \\ ${ }^{3}$ Institute of Applied Physics, Academy of Science of Moldova, Chişinău, Moldova
}

\begin{abstract}
Different reaction mechanisms contribute to the production of light fragments (LF) from nuclear reactions. Available models cannot accurately predict emission of LF from arbitrary reactions. However, the emission of LF is important for many applications, such as cosmic-ray-induced single event upsets, radiation protection, and cancer therapy with proton and heavy-ion beams, to name just a few. The cascade-exciton model (CEM) and the Los Alamos version of the quark-gluon string model (LAQGSM), as implemented in the CEM03.03 and LAQGSM03.03 event generators used in the Los Alamos Monte Carlo transport code MCNP6, describe quite well the spectra of fragments with sizes up to ${ }^{4} \mathrm{He}$ across a broad range of target masses and incident energies. However, they do not predict high-energy tails for LF heavier than ${ }^{4} \mathrm{He}$. The standard versions of CEM and LAQGSM do not account for preequilibrium emission of LF larger than ${ }^{4} \mathrm{He}$. The aim of our work is to extend the preequilibrium model to include such processes. We do this by including the emission of fragments heavier than ${ }^{4} \mathrm{He}$ at the preequilibrium stage, and using an improved version of the Fermi Break-up model, providing improved agreement with various experimental data.
\end{abstract}

\section{Introduction}

Emission of light fragments (LF) from nuclear reactions is an interesting open question. Different reaction mechanisms contribute to their production; the relative roles of each, and how they change with incident energy, mass number of the target, and the type and emission energy of the fragments are not completely understood.

The cascade-exciton model (CEM) [1, 2] version 03.03 and the Los Alamos version of the quarkgluon string model (LAQGSM) [2,3] version 03.03 event generators in the Los Alamos transport code MCNP6 [4] describe quite well the spectra of fragments with sizes up to ${ }^{4} \mathrm{He}$ across a broad range of target masses and incident energies (up to $\sim 5 \mathrm{GeV}$ for CEM and up to $\sim 1 \mathrm{TeV} / \mathrm{A}$ for LAQGSM). However, they do not predict the high-energy tails of LF spectra heavier than ${ }^{4} \mathrm{He}$ well. Most LF with energies above several tens of $\mathrm{MeV}$ are emitted during the precompound stage of a reaction. The standard versions of the CEM and LAQGSM event generators do not account for precompound emission of these heavier LF.

The aim of our work is to extend the precompound model to include such processes, leading to an increase of predictive power of LF-production in MCNP6. This entails upgrading the modified

\footnotetext{
ae-mail: mashnik@lanl.gov
} 
exciton model (MEM) currently used at the preequilibrium stage in CEM and LAQGSM. It will also include expansion and examination of the coalescence and Fermi break-up models used in the precompound stages of spallation reactions within CEM and LAQGSM. Extending our models in this way has provided preliminary results that have much better agreement with experimental data.

\section{Theoretical Background}

These models consider that a reaction begins with the intranuclear cascade, referred to as the INC. The incident particle or nucleus (in the case of LAQGSM) enters the target nucleus and begins interacting with nucleons, scattering off them and also often creating new particles in the process. The incident particle and all newly created particles are followed until they either escape from the nucleus or reach a threshold energy and are then considered "absorbed" by the nucleus.

The preequilibrium stage uses the modified exciton model (MEM) to determine emission of protons, neutrons, and fragments up to ${ }^{4} \mathrm{He}$ from the residual nucleus. In the evaporation stage nucleons in the outer shells of the residual nucleus can "evaporate" off, either singly or as fragments. The CEM evaporation stage is modeled with a modification of Furihata's generalized evaporation model code (GEM2) [5], and can emit light fragments up to ${ }^{28} \mathrm{Mg}$. During and after evaporation, the code looks to see if there is a nuclide with $Z \geq 65$ which is thus fissionable. If this nuclide is randomly determined to fission, the code allows for evaporation from the fission fragments.

There are two models that are not directly part of this linear progression just outlined: coalescence and Fermi break-up. The INC stage only emits nucleons and pions (and other particles, in the case of LAQGSM at high energies), so the coalescence model "coalesces" some of the nucleons produced in the INC into larger fragments by comparing their momenta. If their momenta are similar enough then they coalesce. The current coalescence model can only coalesce up to a ${ }^{4} \mathrm{He}$ fragment, the same as the preequilibrium stage. The Fermi break-up is a very simplified multifragmentation model that is fast and accurate for small atomic numbers; in the standard CEM and LAQGSM models it is used when any nuclide has a mass number less than 13.

More details on the models can be found in Refs. [1-3]. As the MEM uses a Monte-Carlo technique to solve the master equations describing the behavior of the nucleus at the preequilibrium stage (see details in [1]), it is very easy to extend the number of types of possible LF that can be emitted. We have extended the MEM to consider emission of up to 66 types of nucleons and LF, up to ${ }^{28} \mathrm{Mg}$ [6]. As a starting point, for the inverse cross sections, Coulomb barriers, and binding energies of all LF we use the approximations adopted by GEM2 [5].

\section{Results and Conclusion}

Expanding the Fermi break-up model to include heavier LF (up to $A=16$ ) yields increased accuracy for reactions with lighter targets. Figs. 1 and 2 provide examples of calculations by our updated CEM and LAQGSM models, respectively compared to experimental data [7]-[12]. As can be seen, results from the expanded model achieve good agreement with experimental results for these light nuclei.

Expanding the MEM to include heavier LF (up to ${ }^{28} \mathrm{Mg}$ ) yields increased accuracy for reactions on medium and heavy nuclei. Figs. 3 and 4 compare our simulations using the expanded MEM with data by Green et al. [13] and Budzanowski et al. [15].

Similar results for different LF spectra are obtained for several other reactions (see, e.g., [6]).

Our results indicate that expanding the MEM used by the CEM and LAQGSM event generators of MCNP6 to include LF preequilibrium emission significantly increases accuracy of the high-energy spectra compared to experimental data. 

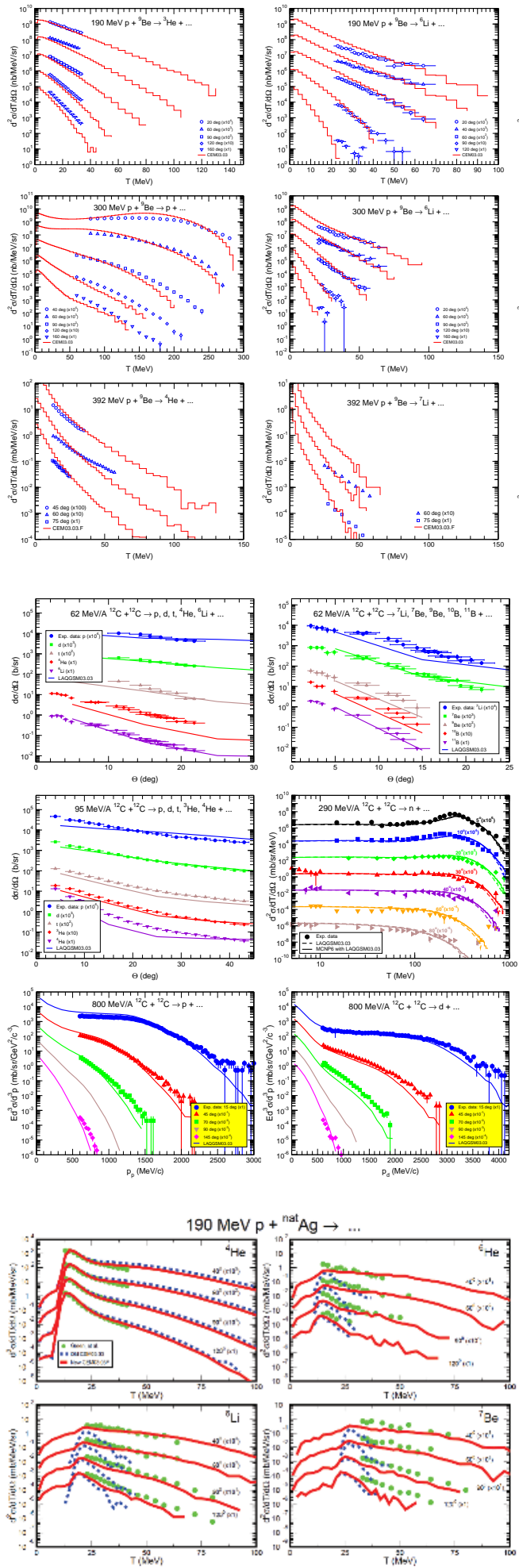
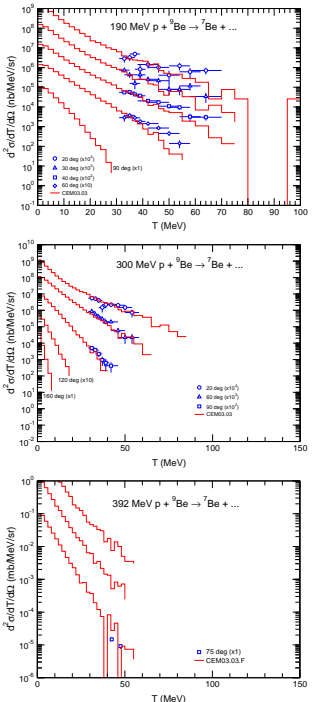

Figure 1. Examples of measured particle and LF double-differential spectra from $\mathrm{p}+{ }^{9} \mathrm{Be}$ at 190,300 , and 392 $\mathrm{MeV}$ [7] (symbols) compared with our CEM results (histograms). All the LF from these reactions are calculated by CEM either using the Fermi break-up model after the INC or as final products (residual nuclei) after the INC and Fermi break-up stages of interactions (Fermi break-up is used for nuclei with $A<13$ instead of using preequilibrium emission and/or evaporation of particles).
Figure 2. Examples of measured particle and LF angular, double-differential, and invariant spectra from ${ }^{12} \mathrm{C}+{ }^{12} \mathrm{C}$ at $62 \mathrm{MeV} / \mathrm{A}$ [8], $95 \mathrm{MeV} / \mathrm{A}$ [9, 10], 290 $\mathrm{MeV} / \mathrm{A}$ [11], and $800 \mathrm{MeV} / \mathrm{A}$ [12] (symbols) compared with LAQGSM03.03 results and calculations by MCNP6 using LAQGSM03.03. All the LF from these reactions are calculated by LAQGSM either using the Fermi break-up model after the INC or as final products (residual nuclei) after the INC and Fermi break-up stages of the interactions (Fermi break-up is used for nuclei with $A<13$ instead of using preequilibrium emission and/or evaporation of particles). Note that our INC does not account for $\alpha$-clustering in ${ }^{12} \mathrm{C}$ and is not well grounded at low energies, therefore the calculated spectra of ${ }^{4} \mathrm{He}$ are under-predicted and generally, the higher the incident energy, the better the agreement with the experimental data.

Figure 3. Examples of measured LF double-differential spectra from $190 \mathrm{MeV} \mathrm{p}+\mathrm{Ag}$ [13] (green circles) compared with results by the extended CEM (solid red lines) and by the older "standard" CEM (blue dashed lines). All the energetic LF heavier than ${ }^{4} \mathrm{He}$ from these reactions are calculated by CEM with the extended preequilibrium model. Similar results are obtained for other LF spectra from this reaction as well as for the $\mathrm{p}+$ $\mathrm{Ag}$ reactions measured by Green et al. at 300 and 480 $\mathrm{MeV}[13,14]$. 

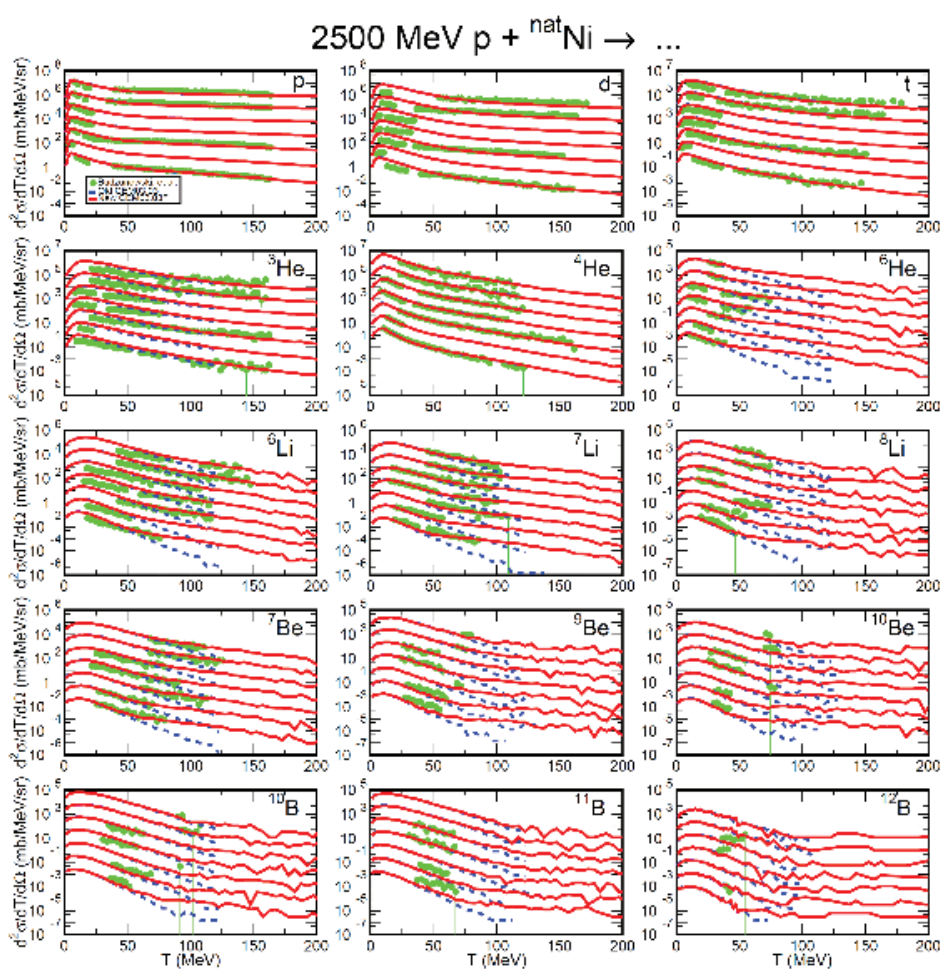

Figure 4. Measured LF double-differential spectra from $2.5 \mathrm{GeV} \mathrm{p}+\mathrm{Ni}$ [15] (green circles) at 15.6, 20, 35, 50, 65, 80, and 100 degrees (multiplied, respectively, by $10^{6}, 10^{5}, 10^{4}, 10^{3}$, $10^{2}, 10^{1}$, and 1) compared with results by the extended CEM (solid red lines) and by the older "standard" CEM (blue dashed lines). Note that all energetic LF heavier than ${ }^{4} \mathrm{He}$ from these reactions are calculated by CEM with the extended preequilibrium model. Similar results are obtained for several other LF spectra from this reaction as well as for the $\mathrm{p}+\mathrm{Ni}$ reactions measured by Budzanowski et al. at 1.2 and $1.9 \mathrm{GeV}$ [15].

We thank Jeremie Dudouet for providing prepublication numerical values of the measurements presented in Ref. [10]. This study was carried out under the auspices of the National Nuclear Security Administration of the U.S. Department of Energy at Los Alamos National Laboratory under Contract No. DE-AC52-06NA253996.

\section{References}

[1] K. K. Gudima et al., Nucl. Phys. A401, 329 (1983).

[2] S. G. Mashnik et al., LANL Report LA-UR-08-2931, Los Alamos (2008); arXiv:0805.0751.

[3] K. K. Gudima et al., LANL Report LA-UR-01-6804, Los Alamos (2001), http://mcnp.lanl.gov/.

[4] T. Goorley et al., Nucl. Technol. 180, 298 (2012).

[5] S. Furihata, Nucl. Instrum. Meth. B 171, 252 (2000).

[6] L. Kerby et al., LANL Report LA-UR-12-24190, Los Alamos (2012), http://mcnp.lanl.gov/.

[7] Y. Uozumi et al., Nucl. Instrum. Meth. A 571, 743 (2007).

[8] M. De Napoli et al., Phys. Med. Biol. 57, 7651 (2012).

[9] J. Dudouet et al., Nucl. Instrum. Meth. A 715, 98 (2013).

[10] J. Dudouet et al., arXiv:1306.0378, June 3, 2013.

[11] Y. Iwata et al., Phys. Rev. C 64, 054609 (2001).

[12] M.-C. Lemaire et al., Lawrence Berkely National Laboratory Report LBL-8463 (1978).

[13] R. E. L. Green et al., Phys. Rev. C 35, 1341 (1987).

[14] R. E. L. Green et al., Phys. Rev. C 29, 1806 (1984).

[15] A. Budzanowski et al., Phys. Rev. C 82, 034605 (2010). 